\title{
Pharmacokinetics and pharmacogenetics of the MEK1/2 inhibitor, selumetinib, in Asian and Western healthy subjects: a pooled analysis
}

\author{
Angela W. Dymond ${ }^{1,2} \cdot$ Cathy Elks $^{3} \cdot$ Paul Martin $^{1,4} \cdot$ David J. Carlile $^{5}$. \\ Gabriella Mariani $^{6}$ - Susan Lovick ${ }^{1,7}$ - Yifan Huang ${ }^{8}$ - Ulrike Lorch ${ }^{9} \cdot$ Helen Brown ${ }^{3}$. \\ Karen $\mathrm{So}^{6}$
}

Received: 12 December 2016 / Accepted: 9 February 2017 /Published online: 10 March 2017

(C) The Author(s) 2017. This article is published with open access at Springerlink.com

\begin{abstract}
Purpose Emerging data on selumetinib, a MEK1/2 inhibitor in clinical development, suggest a possible difference in pharmacokinetics (PK) between Japanese and Western patients. This pooled analysis sought to assess the effect of ethnicity on selumetinib exposure in healthy Western and Asian subjects, and to identify any association between genetic variants in the UGT1A1, CYP2C19 and ABCG2 genes and observed differences in selumetinib PK.

Methods A pooled analysis of data from ten Phase I studies, one in Asian subjects (encompassing Japanese, non-Japanese
\end{abstract}

Electronic supplementary material The online version of this article (doi:10.1007/s00228-017-2217-3) contains supplementary material, which is available to authorized users.

Karen So

karen.so@astrazeneca.com

AstraZeneca, Alderley Park, Macclesfield, Cheshire SK10 4TG, UK

2 Present address: Covance, Early Clinical Biometrics, Leeds, UK

3 AstraZeneca, Personalised Healthcare \& Biomarkers, Innovative Medicines and Early Development Biotech Unit, Darwin Building, 310 Cambridge Science Park, Milton Road, Cambridge CB4 0WG, UK

4 Present address: Sandoz Pharmaceuticals, Holzkirchen, Germany

5 Early Clinical Development, Innovative Medicines and Early Development Biotech Unit, Da Vinci Building, Melbourn, Royston, Hertfordshire SB8 6HB, UK

6 AstraZeneca Global Medicines Development, Da Vinci Building, Melbourn, Royston, Hertfordshire SB8 6HB, UK

7 Present address: Phastar Ltd, London, UK

8 AstraZeneca, Gaithersburg, MD 20878, USA

9 Richmond Pharmacology Ltd, St George's University of London, London SW17 0RE, UK
Asian and Indian Asian subjects) and nine in Western subjects, was conducted. Key findings were derived from the collective exposure data across doses of $25,35,50$ and $75 \mathrm{mg}$ selumetinib; primary variables were dose-normalized AUC and $\mathrm{C}_{\max }$.

Results PK data from 308 subjects (10 studies) were available for the pooled analysis; genetic data from 87 subjects $(3$ studies) were available for the pharmacogenetic analysis. Dose-normalized AUC and $\mathrm{C}_{\max }$ were $35 \%$ (95\% CI: $25-$ $47 \%$ ) and $39 \%$ (95\% CI: $24-56 \%$ ) higher in the pooled Asian group, respectively, compared with Western subjects. PK exposure parameters were similar between the Japanese, non-Japanese Asian and Indian groups. There was no evidence that the polymorphisms assessed in the genes UGT1A1, CYP2C19 and ABCG2 account for observed PK differences.

Conclusions Selumetinib exposure was higher in healthy Asian subjects compared with Western subjects, and these data provide valuable insight for clinicians to consider when treating patients of Asian ethnicity with selumetinib.

Keywords Selumetinib · Asian · Western ·

Pharmacokinetics $\cdot$ Pharmacogenetics

\section{Introduction}

Ethnicity and genetic variability in metabolizing enzymes and transporter proteins can influence the pharmacokinetics (PK) and clinical response to drugs, resulting in variability in the response of individuals from different ethnic groups to standard doses of drugs [1]. This raises the risk of therapeutic failure or adverse drug reactions. Consequently, an assessment of the PK and PD (pharmacodynamic) in key ethnic groups is 
an important consideration in the registration of new medicines, and relevant international guidance has been adopted by the Food and Drug Administration and European Medicines Agency in this context [2].

Selumetinib (AZD6244; ARRY-142886) is an oral, potent and selective, allosteric MEK1/2 inhibitor [3] dosed twicedaily with a short half-life $[4,5]$, currently in development for oncology indications [6-8]. Very limited data are available on the administration of selumetinib in Asian (Japanese, nonJapanese Asian and Indian) patients, although studies that include Asian patients are underway examining selumetinib in combination with the epidermal growth factor receptor inhibitor gefitinib (NCT02025114 [9]), osimertinib (selumetinib up to $50 \mathrm{mg}$ twice-daily [10]) or docetaxel NCT02448290 [11]. Emerging patient PK data indicate that there may be a difference in selumetinib PK between Japanese and Western patients (NCT01605916 [12]), therefore we sought to investigate further the potential impact of ethnicity on selumetinib PK.

The PK of selumetinib have recently been assessed in ten Phase I studies in healthy subjects; these included one study in Asian subjects (Study 86 [NCT01960374]) and nine studies in Western subjects (Studies 66 [NCT01635023], 69 [NCT01974349], 71 [NCT02056392], 78 [NCT02322749], 80 [NCT02238782], 81 [NCT02063204], 82 [NCT02063230], 83 [NCT02093728] and 85 [NCT02046850]). Pooled analysis of selumetinib PK from these studies could further characterize any effects that ethnicity may have on selumetinib PK in Western, Japanese, non-Japanese Asian, and Indian Asian subjects.

Any differences in selumetinib PK between Japanese and Western patients could be influenced by metabolizing enzymes and/or transporters. The cytochrome P450 2C19 (CYP2C19) and UDP glucuronosyltransferase family 1 member A1 (UGT1A1) metabolizing enzymes (encoded by the CYP2C19 and UGT1A1 genes), and the Breast Cancer Resistance Protein (BCRP transporter protein; encoded by the $A B C G 2$ gene) have important roles in drug metabolism and uptake, respectively [13-15]. Since selumetinib is a substrate of these enzymes and transporter protein (unpublished data $^{1}$ ), its metabolism and uptake may be affected, impacting on drug exposure. Studies have shown that these metabolizing enzymes and transporter proteins are polymorphic with allele frequencies that differ between ethnic populations [16-18]. Polymorphisms in genes for metabolizing enzymes and/or transporters among individuals can influence the efficacy and toxicity of some anti-cancer therapies [19]. Hence, it is important to explore whether genetic variation could account for any differences observed in the PK of selumetinib. The precise quantitative contributions of metabolizing enzymes to

\footnotetext{
$\overline{{ }^{1} \text { Report nos. Be } 000021-21 ~ a n d ~ D 6244 ~ K M N 036, ~ d a t a ~ o n ~ f i l e, ~ A s t r a Z e n e c a, ~}$ Cheshire, UK
}

the clearance of selumetinib are not known but it is evident that CYP3A4 and CYP2C19 [20] as well as direct conjugation are involved.

These analyses may provide valuable insight into dose selection in these ethnically diverse populations, and could guide future clinical studies in patients.

\section{Subjects and methods}

\section{Study objectives}

This pooled analysis of PK and pharmacogenetic data from Phase I, single-dose studies of selumetinib $(10 \mathrm{mg}$ and $25 \mathrm{mg}$ capsules were used to deliver $25,35,50$ and $75 \mathrm{mg}$ dose levels) was conducted in healthy subjects of Asian or Western ethnicity (Online Resource 1). The primary objective of the analysis was to characterize any effects of ethnicity on the exposure of single-dose selumetinib in healthy subjects and to assess dose proportionality in Western and Asian subjects. A secondary objective was to explore whether genetic variants of the CYP2C19, UGT1A1 and ABCG2 genes might contribute to any selumetinib PK differences observed between Asian and Western subjects. All the studies included in the pooled analysis were conducted in accordance with the ethical principles outlined in the Declaration of Helsinki and the International Council on Harmonization Good Clinical Practice. Written informed consent was obtained from all subjects prior to any study-related procedures.

\section{Database construction}

The pooled analysis database was constructed using data collected in 10 studies (Online Resource 1). PK data were taken from Study 86 (Asian study) and Studies 66, 69, 71, 78, 80, $81,82,83$ and 85 (Western studies), while pharmacogenetic data were obtained from Study 86 (Asian study) and Studies 66 and 83 (Western studies). Informed consent for genetic assessment was as follows: Study 66, 21 of 27 volunteers gave consent (one of these volunteers discontinued treatments); Study 83, all 26 volunteers gave consent; Study 86, 45 of 72 volunteers gave consent.

Study 86 (Asian study) was a single-center, open-label, dose-escalation study conducted in the UK to assess the safety, tolerability and single-dose PK characteristics of selumetinib in healthy Japanese subjects (first-generation, born in Japan; expatriate of Japan for $<5$ years), nonJapanese Asian subjects (first-generation, born in an Asian country other than Japan, but not India; expatriate of their country of origin for $<5$ years) or Indian ethnicity (first-generation, born in the Indian subcontinent; expatriate of their country of origin for $<5$ years). 
Selumetinib dosing started at $25 \mathrm{mg}$, with escalation planned to a dose level with exposure equivalent to (and not exceeding) the maximum dose of $75 \mathrm{mg}$ permitted in Western healthy subjects. This maximum dose and associated PK exposure limits were set by AstraZeneca safety committee. In the Western studies, subjects were classified as being of White or Black ethnicity; one subject from Study 66 was identified as Asian, but was excluded from the pooled analysis due to a lack of further information regarding his ethnicity.

Further inclusion criteria for the database included fasted subjects dosed with the selumetinib capsule formulation used for Phase III. This meant exclusion of data from other oral formulations used in study 78 and intravenous formulation used in study 80. Data were included solely from study arms where there was an absence of other treatments (e.g. rifampicin, fluconazole) and from subjects with normal renal and hepatic function.

\section{Pooled analysis of selumetinib exposure and dose proportionality}

A merged dataset containing subject identifier, noncompartmental PK parameters and demographics was constructed. The non-compartmental PK parameters analyzed were: area under the plasma concentration-time curve from time zero to infinity (AUC); area under the plasma concentration-time curve from time $0-12 \mathrm{~h}\left(\mathrm{AUC}_{0-12}\right)$; maximum observed plasma concentration $\left(\mathrm{C}_{\max }\right)$; time to $\mathrm{C}_{\max }$ $\left(\mathrm{t}_{\max }\right)$; elimination half-life $\left(\mathrm{t}_{1 / 2}\right)$; apparent oral clearance $(\mathrm{CL} / \mathrm{F})$.

The PK parameters AUC, $\mathrm{AUC}_{0-12}$ and $\mathrm{C}_{\max }$ were normalized (abbreviated as DN) for each subject, by dividing the specific PK parameter by the nominal dose (in mg units) and for dose per kg of body weight (abbreviated as DWN) using the following formula: PK parameter value / (nominal dose in $\mathrm{mg}$ units / subjects baseline body weight in kg units).

The majority of subjects in the Western studies were of White ethnicity, thus this formed the reference category for comparison. Statistical comparisons of selumetinib PK were undertaken between the following ethnic groups: Western vs All Asian; White vs Black; White vs All Asian; White vs Japanese; White vs nonJapanese Asian; White vs Indian. The comparisons were performed by fitting multivariate models to the PK parameters of interest (e.g. dose-normalized AUC and $\mathrm{C}_{\max }$ ), with a stepwise backward selection of the covariates including ethnicity, age, sex, and weight.

Dose-exposure proportionality for $\mathrm{AUC}$ and $\mathrm{C}_{\max }$ was assessed for non-Japanese Asian subjects using data from Study 86, and for the pooled analysis Western group by applying the Power model to data from the pooled analysis: $\log (\mathrm{Y})=\alpha+\beta \cdot \log ($ Dose $)+\varepsilon$, where $\mathrm{Y}=\mathrm{AUC}$ or $\mathrm{C}_{\max }$. Intercept and slope estimates (including point estimate, standard error, and $90 \%$ confidence interval $[\mathrm{CI}]$ ) were provided for each parameter, and dose proportionality plots were generated for AUC and $\mathrm{C}_{\max }$. All statistical analyses were performed using SAS version 9.2.

\section{Bioanalytical methods}

Details of the bioanalytical methods are described in Online Resource 2.

\section{Analysis of selumetinib pharmacogenetics}

The pharmacogenetics component of the pooled analysis sought to explore whether polymorphisms in the CYP2C19, UGTIAI and $A B C G 2$ genes were associated with differences in selumetinib PK parameters following drug administration. Five of the six genetic variants analyzed $(C Y P 2 C 19 * 2, C Y P 2 C 19 * 3$, $C Y P 2 C 19 * 17, U G T 1 A 1 * 6, A B C G 2421 C>A$ ) were single nucleotide polymorphisms while one genetic variant was a tandem repeat $(U G T 1 A 1 * 28$.) Genotyping of the variants was performed using a Luminex CYP2C19 assay (for CYP2C19*2, $C Y P 2 C 19 * 3$ and $C Y P 2 C 19 * 17$ ), Taqman assays (for $U G T 1 A 1 * 6$ and $A B C G 2421 C>A$ ), and fragment analysis (for $U G T 1 A 1 * 28)$.

Each genetic variant was tested for any association with dose-normalized AUC, $\mathrm{AUC}_{0-12}$ or $\mathrm{C}_{\max }$, using linear regression under an additive genetic model (i.e. phenotypic response per additional copy of the variant allele, where 0 , 1 or 2 copies can be present at each locus). For each PK parameter Bonferroni's correction was used to control for multiple testing when assessing statistical significance. A $p$ value below 0.008 ( $p<0.05$ with Bonferoni correction for 6 tests) was considered statistically significant. Given the variation in frequency of the variant alleles, analyses were performed stratified by ethnic group (Asian, White, Black) across the studies. A combined estimate across populations was obtained using random effects meta-analysis to allow for between-population heterogeneity.

Genotype distributions were tested for deviation from Hardy-Weinberg equilibrium using Chi-squared tests. All pharmacogenetic analyses were performed using either StataSE version 14.1 or R statistical software version 3.2.2.

\section{Safety and tolerability}

Safety and tolerability findings obtained from Study 86 were summarized and compared descriptively to the safety and tolerability findings from the studies in Western subjects. Additional safety and tolerability data for the Western studies are reported in detail elsewhere and include references [20-22]. 
Table 1 Summary of selumetinib PK parameters by ethnicity

\begin{tabular}{|c|c|c|c|c|c|c|c|}
\hline Parameter ${ }^{*}$ & $\begin{array}{l}\text { White } \\
(N=149)\end{array}$ & $\begin{array}{l}\text { Black } \\
(N=87)\end{array}$ & $\begin{array}{l}\text { Japanese } \\
(N=27)\end{array}$ & $\begin{array}{l}\text { non-Japanese Asian } \\
(N=36)\end{array}$ & $\begin{array}{l}\text { Indian } \\
(N=9)\end{array}$ & $\begin{array}{l}\text { Western } \\
(N=236)\end{array}$ & $\begin{array}{l}\text { All Asian } \\
(N=72)\end{array}$ \\
\hline DN AUC, ng.h/mL/mg & $51.9(23.9)^{\dagger}$ & $53.4(26.4)$ & $84.8(21.0)$ & $78.9(25.4)$ & $69.5(16.7)$ & $52.4(24.9)^{\ddagger}$ & $79.8(23.6)$ \\
\hline $\mathrm{DN} \mathrm{AUC}_{(0-12)}, \mathrm{ng} \cdot \mathrm{h} / \mathrm{mL} / \mathrm{mg}$ & $42.7(24.2)$ & $43.3(26.7)$ & $65.7(24.1)$ & $61.1(24.6)$ & $53.5(19.6)$ & $42.9(25.1)$ & $61.7(24.6)$ \\
\hline $\mathrm{DN} \mathrm{C}_{\max }, \mathrm{ng} / \mathrm{mL} / \mathrm{mg}$ & $17.7(33.2)$ & $16.9(39.6)$ & $28.7(31.0)$ & $24.3(32.3)$ & $28.1(16.0)$ & $17.4(35.6)$ & $26.3(30.9)$ \\
\hline$t_{\max }, h$ & $1.0(1,4)$ & $1.0(1,4)$ & $1.0(1,3)$ & $1.6(1,4)$ & $1.0(1,2)$ & $1.0(1,4)$ & $1.0(1,4)$ \\
\hline$t_{1 / 2}, h$ & $9.3(3.11)^{\dagger}$ & $8.7(2.51)$ & $11.5(3.96)$ & $9.8(2.58)$ & $9.5(3.31)$ & $9.1(2.91)^{\ddagger}$ & $10.4(3.32)$ \\
\hline $\mathrm{CL} / \mathrm{F}, \mathrm{L} / \mathrm{h}$ & $19.9(5.40)^{\dagger}$ & $19.5(5.71)$ & $12.0(2.56)$ & $13.0(2.94)$ & $14.6(2.32)$ & $19.7(5.51)^{*}$ & $12.8(2.81)$ \\
\hline
\end{tabular}

*AUC, $\mathrm{AUC}_{(0-12)}$ and $\mathrm{C}_{\max }$ are presented as geometric mean $(\% \mathrm{CV}) ; \mathrm{t}_{\max }$ is presented as median (min, max); $\mathrm{t}_{1 / 2}, \mathrm{CL} / \mathrm{F}$ and are presented as arithmetic mean (SD); ${ }^{\dagger} N=148 ;{ }^{\ddagger} N=235$

$\mathrm{AUC}$, area under the plasma concentration time curve to infinity; $\mathrm{AUC}_{(0-12)}, \mathrm{AUC}$ from 0 to $12 \mathrm{~h} ; C L / F$, apparent oral clearance; $C_{\text {max }}$, maximum observed plasma concentration; $D N$, dose normalized; $t_{\max }$, time to maximum plasma concentration; $t_{1 / 2}$, elimination half-life; $S D$, standard deviation

\section{Results}

\section{Demographics and characteristics}

Details of the 10 studies included in the pooled analysis are summarized in Online Resource 1. Selumetinib was administered as a single $25 \mathrm{mg}$ dose (Study 83), a $50 \mathrm{mg}$ dose (Studies 81 and 82 ) or a $75 \mathrm{mg}$ dose (Studies 66, 69, 71, 78, 80 and 85). In Study 86 (Asian study), administered selumetinib doses were 25 and $35 \mathrm{mg}$ in Japanese subjects, 25,35 or $50 \mathrm{mg}$ in non-Japanese Asian subjects, and $25 \mathrm{mg}$ in Indian subjects.

Baseline demographics and characteristics are summarized by selumetinib dose in Online Resource 3. Data from 308 subjects $(25 \mathrm{mg}, n=53 ; 35 \mathrm{mg}, n=27 ; 50 \mathrm{mg}, n=38$; $75 \mathrm{mg}, n=190$ ) across the 10 studies were included in the pooled analysis. The mean age of subjects was similar across each dose cohort, ranging from 28.5 years in the $35 \mathrm{mg}$ dose group to 41.0 years in the $50 \mathrm{mg}$ dose group. In all dose groups, the majority of subjects were male accounting for $87-100 \%$ of the study populations. White and Black subjects

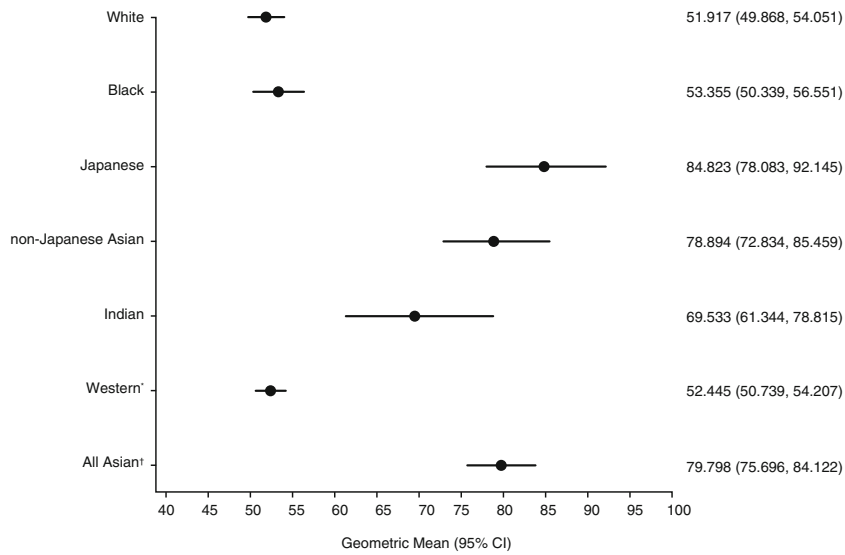

Fig. 1 Forest plots of dose-normalized a) AUC and b) $C_{\max }$, by ethnicity. ${ }^{*}$ White and Black subjects; ${ }^{\dagger} J a p a n e s e$, non-Japanese Asian and Indian subjects. Data units are: $\mathrm{ng} . \mathrm{h} / \mathrm{mL} / \mathrm{mg}$ for AUC; $\mathrm{ng} / \mathrm{mL} / \mathrm{mg}$ for $\mathrm{C}_{\max }$. White, $N=148$ for AUC and $N=149$ for $\mathrm{C}_{\max }$; Black, $N=87$; were more prevalent in the $25 \mathrm{mg}$ and $75 \mathrm{mg}$ dose groups compared with Asian subjects. Japanese subjects were the most prevalent in the $35 \mathrm{mg}$ group, and non-Japanese Asian subjects were the most prevalent in the $50 \mathrm{mg}$ group.

A total of 91 subjects across Studies $66(n=20), 83$ $(n=26)$ and $86(n=45)$ were available for pharmacogenetic assessment. Given observed differences in allele frequencies in the variants being studied between South and East Asian populations, and the potential for population stratification to confound these analyses, Indian Asian subjects $(n=3)$ from the Asian subsample in Study 86 were excluded. As there was no further information on the Asian subject from Study 66 regarding ethnicity, this subject was also excluded, leaving a total of 87 subjects (42 East Asians, 21 Black or African American and 24 White) for the pharmacogenetic analyses.

\section{Selumetinib PK, by ethnicity}

In studies where increased selumetinib exposure was anticipated, a dose lower than $75 \mathrm{mg}$ was used to ensure that the

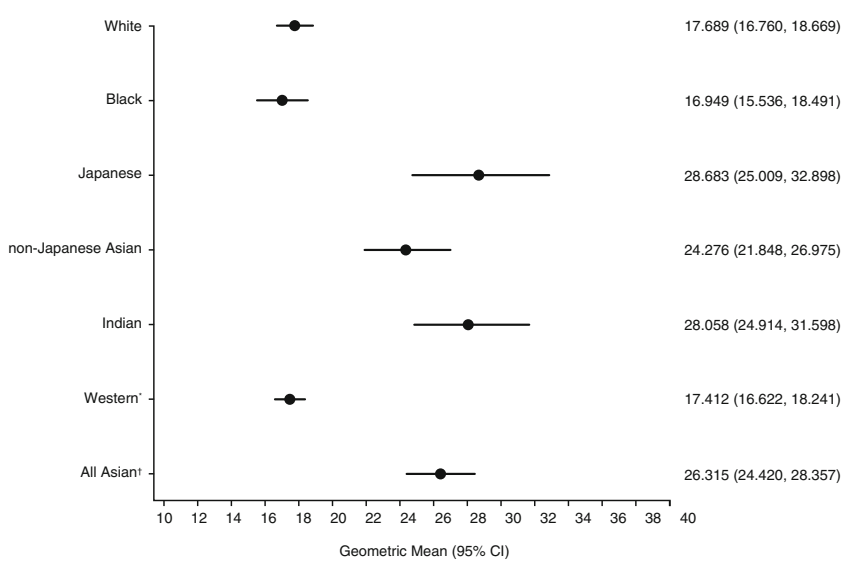

Japanese, $N=27$; non-Japanese Asian, $N=36$; Indian, $N=9$; Western, $N=235$ for AUC and $N=236$ for $\mathrm{C}_{\text {max }}$; All Asian, $N=72$. AUC, area under the plasma concentration time curve to infinity; $\mathrm{C}_{\max }$, maximum plasma concentration; $\mathrm{DN}$, dose normalized 
Table 2 Dose-normalized AUC and $\mathrm{C}_{\max }$, statistical comparisons between ethnic groups

\begin{tabular}{|c|c|c|c|c|c|c|}
\hline \multirow[t]{2}{*}{ Parameter } & \multicolumn{3}{|l|}{ DN AUC* } & \multicolumn{3}{|l|}{$\mathrm{DN} \mathrm{C}_{\max }$} \\
\hline & $\begin{array}{l}\text { Geometric least } \\
\text { squares mean }\end{array}$ & Difference $^{\dagger}$ & $95 \% \mathrm{CI}$ & $\begin{array}{l}\text { Geometric least } \\
\text { squares mean }\end{array}$ & Difference $^{\dagger}$ & $95 \% \mathrm{CI}$ \\
\hline All Asian $(n=72)$ vs Western $(n=236)$ & 4.284 vs 3.982 & 1.353 & $1.246,1.469$ & 3.205 vs 2.877 & 1.388 & $1.235,1.560$ \\
\hline Black $(n=87)$ vs White $(n=149)$ & 3.989 vs 3.975 & 1.014 & $0.951,1.081$ & 2.847 vs 2.907 & 0.942 & $0.860,1.032$ \\
\hline Japanese $(n=27)$ vs White $(n=149)$ & 4.384 vs 3.975 & 1.505 & $1.354,1.673$ & 3.280 vs 2.907 & 1.453 & $1.251,1.687$ \\
\hline non-Japanese Asian $(n=36)$ vs White $(n=149)$ & 4.292 vs 3.975 & 1.373 & $1.244,1.516$ & 3.088 vs 2.907 & 1.198 & $1.042,1.377$ \\
\hline Indian $(n=9)$ vs White $(n=149)$ & 4.177 vs 3.975 & 1.223 & $1.034,1.447$ & 3.247 vs 2.907 & 1.405 & $1.108,1.781$ \\
\hline
\end{tabular}

*Western, $n=235$; White, $n=148 ;{ }^{\dagger}$ Point estimate: adjusted ratio of geometric means; for each comparison, a value above one implies an increase in exposure versus the comparator

Data from the following studies: 66 [NCT01635023], 69 [NCT01974349], 71 [NCT02056392], 78 [NCT02322749], 80 [NCT02238782], 81 [NCT02063204], 82 [NCT02063230], 83 [NCT02093728], 85 [NCT02046850] and 86 [NCT01960374]

Data units are: $\mathrm{ng} . \mathrm{h} / \mathrm{mL} / \mathrm{mg}$ for $\mathrm{AUC} ; \mathrm{ng} / \mathrm{mL} / \mathrm{mg}$ for $\mathrm{C}_{\max }$

$A U C$, area under the plasma concentration time curve to infinity; $C I$, confidence interval; $C_{\max }$, maximum plasma concentration; $D N$, dose normalized

mean selumetinib exposure would not exceed the exposure limit. Exposure data from all ethnicity groups under investigation (i.e. Whites, Blacks, Japanese, non-Japanese Asian and Indians) were only available at the $25 \mathrm{mg}$ dose of selumetinib, therefore the use of dose-normalized exposure data across the collective range of doses tested $(25,35,50$ and $75 \mathrm{mg})$ better reflected the exposures experienced in the clinic (therapeutic dose and any dose reductions).

In the pooled analysis including all selumetinib doses, the geometric means (geomeans) for dose-normalized AUC and AUC $_{(0-12)}$ were higher in All Asian subjects (79.8 and $61.7 \mathrm{ng} . \mathrm{h} / \mathrm{mL} / \mathrm{mg}$, respectively) compared with Western subjects (52.4 and 42.9 ng.h $/ \mathrm{mL} / \mathrm{mg}$ ) (Table 1). Of the Asian subcategories, the geomean dose-normalized AUC and $\mathrm{AUC}_{(0-}$ 12) values for selumetinib were generally similar among Indian
(69.5 and $53.5 \mathrm{ng} . \mathrm{h} / \mathrm{mL} / \mathrm{mg}$ ), non-Japanese Asian (78.9 and $61.1 \mathrm{ng} . \mathrm{h} / \mathrm{mL} / \mathrm{mg}$ ) and Japanese ( 84.8 and $65.7 \mathrm{ng} . \mathrm{h} / \mathrm{mL} / \mathrm{mg}$ ) subjects, although there was a slight increase if comparing that of the Japanese subjects with the Indian subjects. In the Western sub-categories, values were similar among White (51.9 and $42.7 \mathrm{ng} . \mathrm{h} / \mathrm{mL} / \mathrm{mg}$ ) and Black (53.4 and $43.3 \mathrm{ng} . \mathrm{h} /$ $\mathrm{mL} / \mathrm{mg}$ ) subjects (Table 1). Geomeans for dose-normalized $\mathrm{C}_{\max }$ followed a similar pattern to $\mathrm{AUC}$ and $\mathrm{AUC}_{(0-12)}$ across these ethnic groups (Table 1).

In terms of the percentage difference in exposure of all selumetinib doses, geomeans for dose-normalized AUC and $\mathrm{C}_{\max }$ were higher in the All Asian group compared with the Western group (Fig. 1), by 35\% (90\% CI: $25-47 \%$ ) and $39 \%$ (90\% CI: 24-56\%), respectively (Table 2). Of the Asian race sub-categories investigated, Japanese showed the greatest

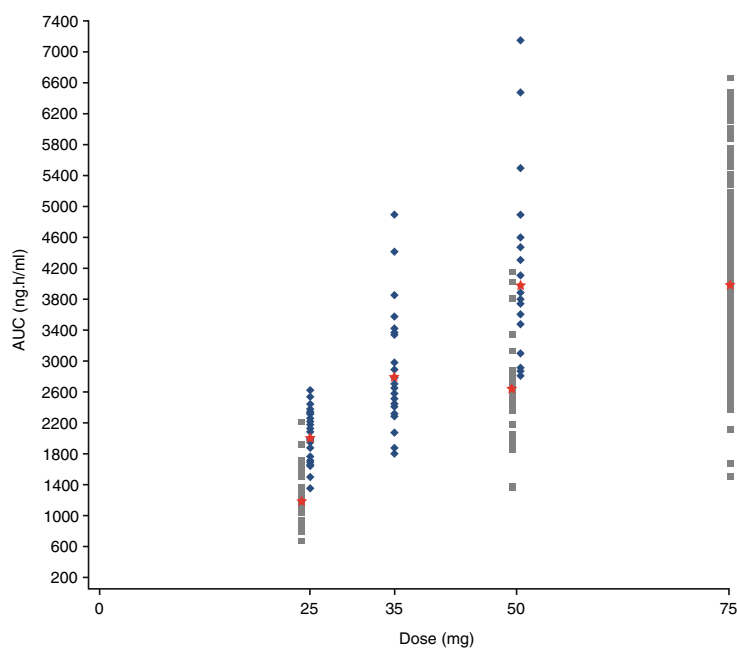

Fig. 2 Scatter plot of a) AUC and b) $C_{\max }$ against dose, by ethnicity. ${ }^{*}$ White and Black subjects; ${ }^{\dagger}$ Japanese, non-Japanese Asian and Indian subjects. Western and All Asian data points at the 25 and $50 \mathrm{mg}$ doses

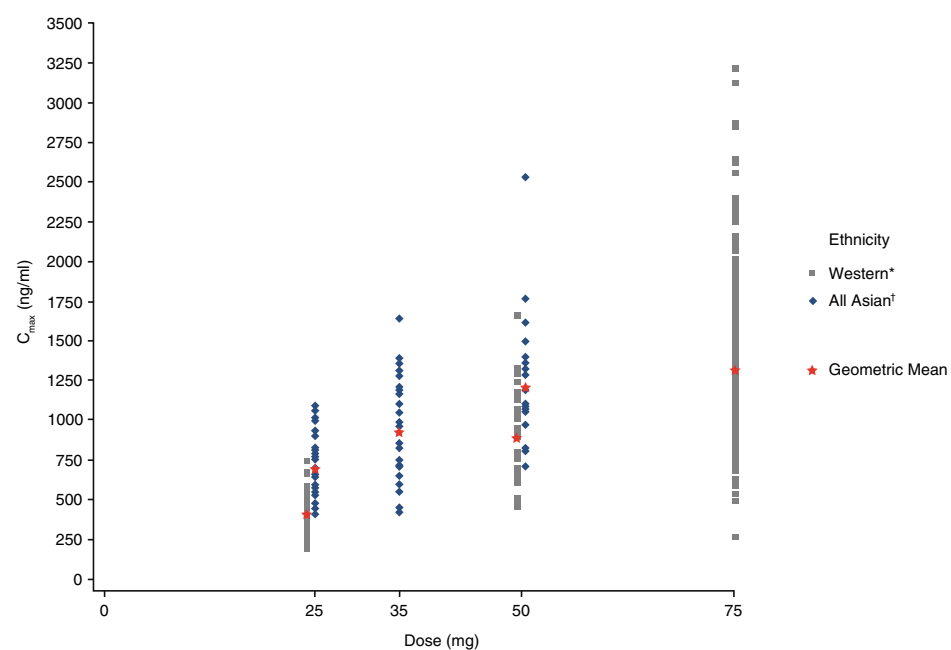

are staggered to allow greater visual clarity. AUC, area under the plasma concentration time curve to infinity; $\mathrm{C}_{\max }$, maximum plasma concentration 

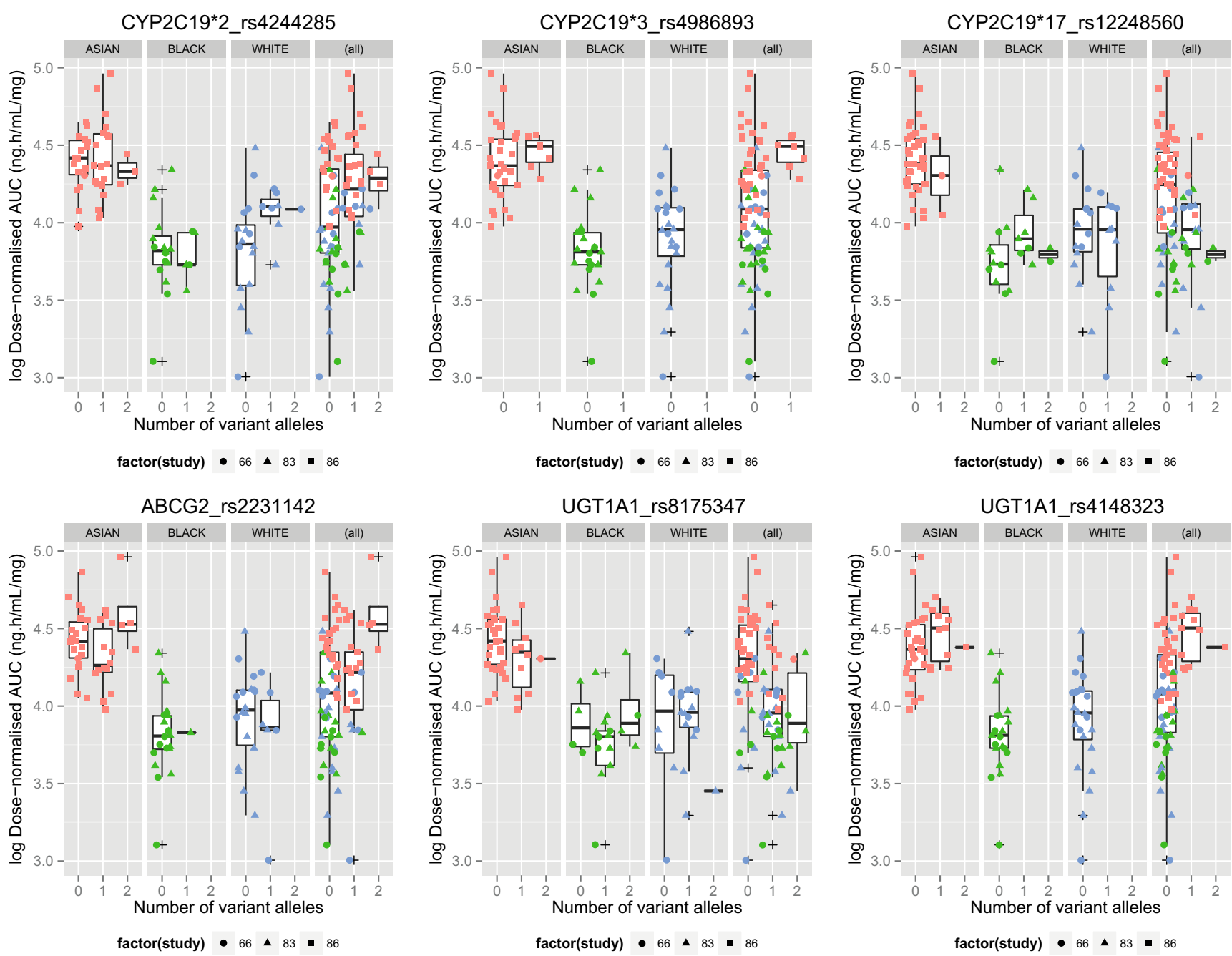

Fig. 3 Dose-normalized AUC in genetic variants of CYP2C19, UGT1A1 and ABCG2, by ethnicity. AUC, area under the plasma concentration time curve to infinity

increase in geomeans for dose-normalized AUC compared to White by 51\% (95\% CI: 35-67\%), followed by non-Japanese Asian (37\% with 95\% CI: 24-52\%) then Indian (22\% with 95\% CI: 3-45\%) (Table 2). A different ordering was detected for $\mathrm{C}_{\max }$ : Japanese (45\% with $95 \%$ CI: $25-69 \%$ ), followed by Indian (41\% with 95\% CI: 11-78\%) then non-Japanese Asian (20\% with 95\% CI: 4-38\%) compared to White (Table 2). Among the Asian subjects, PK parameters were generally similar between the Japanese, non-Japanese Asian and Indian groups, with differences in the geomeans for dose-normalized AUC and dose-normalized $\mathrm{C}_{\max }$ ranging from 2 to $18 \%$ when comparing non-Japanese Asian and Indian against Japanese (Fig. 1, Table 1). No difference in dose-normalized AUC or $\mathrm{C}_{\max }$ was observed between White and Black subjects (Table 2).

Higher geomean exposures were detected in the All Asian subjects compared to Western subjects when AUC (5229 vs $4126 \mathrm{ng} . \mathrm{h} / \mathrm{mL} / \mathrm{mg} / \mathrm{kg}$ ), $\mathrm{AUC}_{(0-12)}$ (4045 vs $3372 \mathrm{ng} . \mathrm{h} / \mathrm{mL} /$ $\mathrm{mg} / \mathrm{kg}$ ) and $\mathrm{C}_{\max }(1724 \mathrm{vs} 1369 \mathrm{ng} / \mathrm{mL}$ ) were normalized by dose per kg of bodyweight (Online Resource 4).
This study showed that the median $\mathrm{t}_{\max }$ of selumetinib peaked at 1.0 to $1.6 \mathrm{~h}$ for all ethnic groups while the arithmetic mean terminal $t_{1 / 2}$ value was slightly higher in All Asian subjects (10.4 h) compared with Western subjects $(9.1 \mathrm{~h})$ (Table 1).

The ANOVA stepwise model fitting procedure identified weight and ethnicity as the key covariates associated with exposure, with age and sex not showing a clear association with exposure. Overall, the PK variability looked similar at all doses of selumetinib, and in each ethnic group.

\section{Selumetinib dose-exposure proportionality, by ethnicity}

Scatter plots for individual and geomean $\mathrm{AUC}$ and $\mathrm{C}_{\max }$ versus selumetinib dose enabled visual assessment of dose-exposure proportionality (Fig. 2). Approximate dose proportionality was observed between single doses of 25 to $75 \mathrm{mg}$ selumetinib in the Western group, whilst approximate dose proportionality between 25 to $50 \mathrm{mg}$ selumetinib was observed in the nonJapanese Asian group (Online Resource 5). 

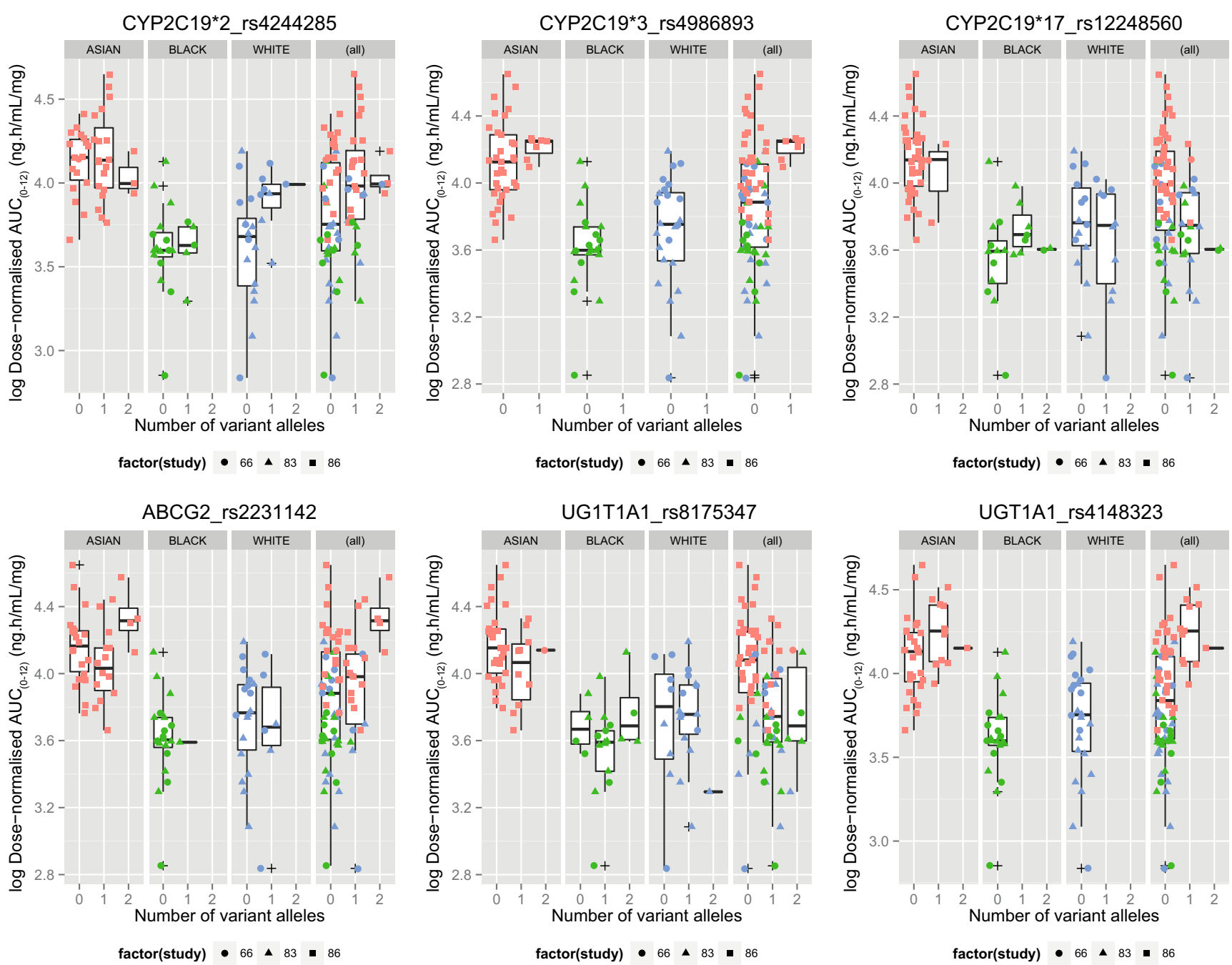

Fig. 4 Dose-normalized $\mathrm{AUC}_{(0-12)}$ in genetic variants of CYP2C19, UGT1A1 and $A B C G 2$, by ethnicity. $\mathrm{AUC}_{(0-12)}$, area under the plasma concentration time curve from 0 to $12 \mathrm{~h}$

Among the Asian subjects, dose proportionality was statistically analyzed only for the non-Japanese Asian group, since this was the only cohort who received three selumetinib dose levels in Study 86. In this non-Japanese Asian group, statistical dose-exposure proportionality was confirmed for AUC over the dose range studied of $25-50 \mathrm{mg}$ (slope estimate 0.99 with $90 \%$ CI: $0.760-1.229$; AUC values completely within the critical region of $0.678-1.322$ ), although not for $\mathrm{C}_{\max }$ (slope estimate 1.017 with $90 \%$ CI: $0.708-1.326$ ). As $\mathrm{C}_{\max }$ was slightly greater than dose proportional, logtransformed $\mathrm{C}_{\max }$ was also analyzed. This confirmed that the increases in $\mathrm{C}_{\max }$ from 25 to $35 \mathrm{mg}$, and from 25 to $50 \mathrm{mg}$, were not directly dose proportional, as the $90 \%$ CIs were just outside the critical region $(0.80-1.25)$. In the Western group, statistical dose-exposure proportionality was observed for $\mathrm{Ln}$ $\mathrm{C}_{\max }$ (slope estimate 1.056 with 95\% CI: 0.924-1.189) and for Ln AUC (slope estimate 1.099 with 95\% CI: 1.005-1.192) where the critical region is $0.797-1.203$ (Online Resource 5).

\section{Selumetinib pharmacogenetics, by ethnicity}

The distribution of genetic variants for subjects included in the pharmacogenetic analysis, in the Asian, White and Black groups, is summarized in Online Resource 6. Allele frequencies varied between ethnic groups but there was no significant deviation from the genotype frequencies expected under Hardy-Weinberg equilibrium within each population $(p>0.05)$. None of the polymorphisms in the CYP2C19, UGT1A1 and ABCG2 genes showed any association with dose-normalized values for $\mathrm{AUC}$ (Fig. 3), $\mathrm{AUC}_{0-12}$ (Fig. 4) or $\mathrm{C}_{\max }$ (Fig. 5) (Online Resource 7) in any ethnic group after accounting for multiple testing. When subjects of all ethnicities were considered together, the variation of PK parameters between individuals could not be explained by the genetic variants under investigation (Online Resource 8). 

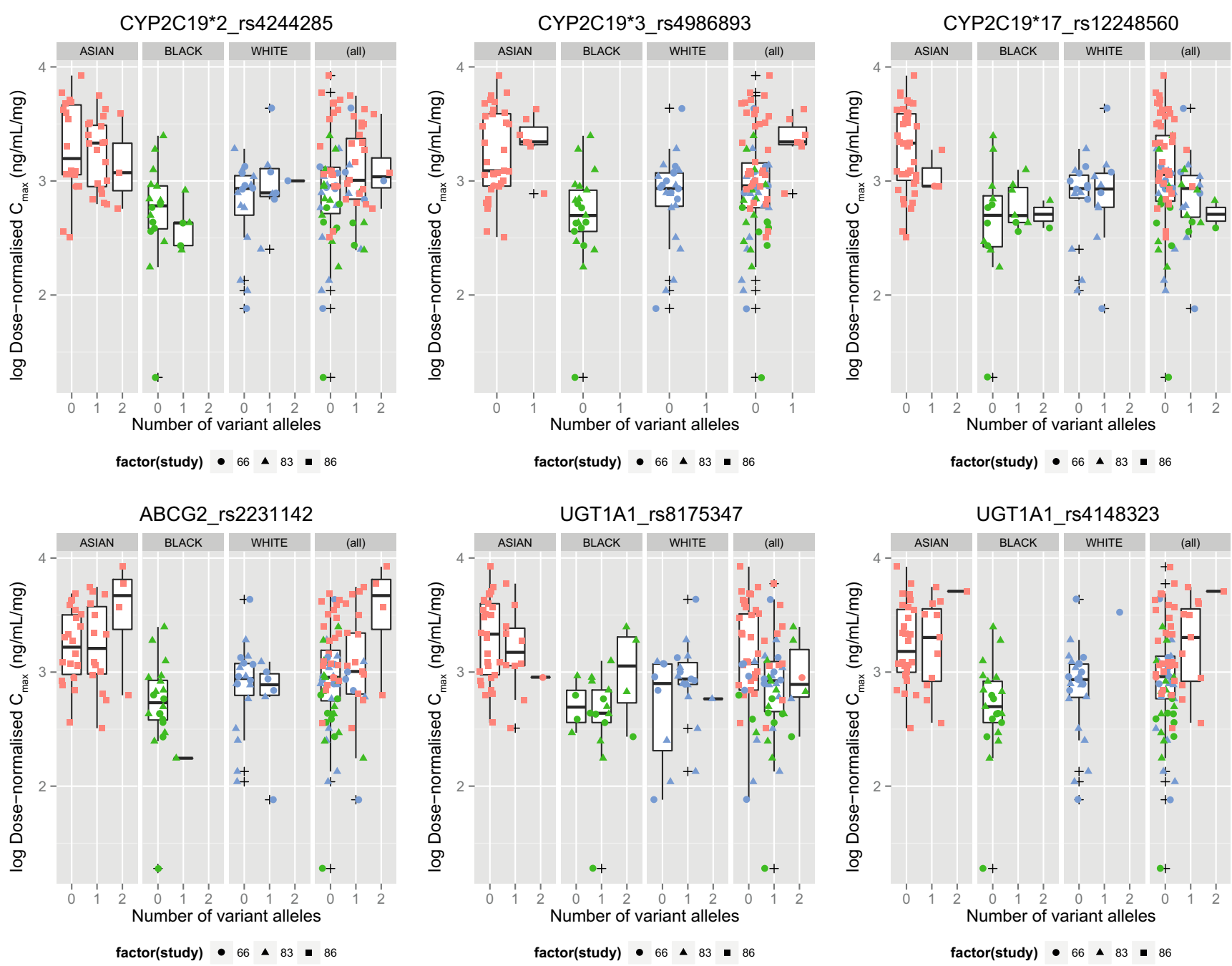

Fig. 5 Dose-normalized $\mathrm{C}_{\max }$ in genetic variants of $C Y P 2 C 19$, UGT1A1 and $A B C G 2$, by ethnicity. $\mathrm{C}_{\max }$, maximum plasma concentration

\section{Safety and tolerability}

In Study 86 (Asian study), there were no safety or tolerability concerns at any dose level $(25,35$ and $50 \mathrm{mg})$; no subject experienced a serious adverse event (AE) and no $\mathrm{AE}$ led to the discontinuation of selumetinib. The number of AEs was highest in the non-Japanese Asian subject group with $50 \mathrm{mg}$ selumetinib while similar numbers of events were reported in each ethnic group at the 25 and $35 \mathrm{mg}$ doses. However, there were no Japanese or Indian subjects who were dosed at $50 \mathrm{mg}$ to make comparisons between the Asian sub-categories. Of the 21 AEs reported, 14 were deemed related to selumetinib by the Investigator. All the AEs were of mild severity except for a single incidence of moderate nausea in a subject who was also experiencing vomiting (mild) at the same time.

These data are consistent with the studies of selumetinib in Western healthy subjects [20-22]. In these studies, there were no serious AEs in Western healthy subjects receiving selumetinib and the majority of AEs were mild and generally considered unrelated to selumetinib. No subjects discontinued selumetinib due to an $\mathrm{AE}$.

\section{Discussion}

This pooled analysis of selumetinib PK and pharmacogenetic findings in Asian and Western healthy subjects explored the impact of ethnicity on selumetinib exposure. The findings provide insight into potential selumetinib dose selection in patients and could be used to guide future studies. The data was analyzed by comparison of exposures in different ethnicities of un-adjusted and dose-normalized PK parameters. This was considered sufficient to provide the required understanding. It is acknowledged that a population PK approach would also have been an appropriate approach. The same bioanalytical method was used for all studies to minimize variability and possible bias in the evaluation. Only data obtained from subjects receiving the same oral formulation as 
used in Phase III studies were included in the analysis again with intent to minimize variability and possible bias due to formulation or route of administration.

Notably, higher exposures were detected in the Asian subjects compared with Western subjects when AUC and $\mathrm{C}_{\max }$ were normalized for dose only, and when normalized by dose per kg of bodyweight. This suggests that higher exposures in Asian subjects are not the result of bodyweight differences alone. Moreover, dose-normalized selumetinib exposure was approximately 35-39\% higher in Asian subjects (Japanese, non-Asian Japanese or Indian) compared with Western subjects (White or Black) following a single selumetinib dose in the range of 25 to $75 \mathrm{mg}$. This finding will be taken into consideration when treating patients of Asian ethnicity as the current therapeutic dose is $75 \mathrm{mg}$ twice-daily for all patients. When data are available from patients, safety/exposure relationships for the different ethnicities can be explored. In addition, the apparent differences from the current study will be considered alongside safety, efficacy and PK in patient studies to determine suitable dose levels and regimes. The small departure from direct dose proportionality observed in this study is not considered to be clinically relevant.

Overall, the generally similar PK exposure between the Japanese, non-Japanese Asian and Indian groups suggest that selumetinib exposure is likely to be similar across Asian populations. Similar exposure was observed in the White and Black populations with no statistically significant differences noted in PK findings; however, it should be noted that the studies included in this retrospective pooled analysis were not initially designed to investigate differences in exposure between White and Black populations.

Genetic variants in the CYP2C19, UGT1A1 and ABCG2 genes did not appear to account for observed PK differences between individuals, suggesting that other genetic or nongenetic factors may be involved, or that there was not enough power to detect such associations.

There were no safety or tolerability concerns in Asian subjects in Study 86; this is similar to the case in the Western studies [20-22]. Furthermore, preliminary data from the TATTON study, which includes Asian patients, indicated no safety concerns in patients who received selumetinib (escalated to a dose of $50 \mathrm{mg}$ twice-daily) in combination with osimertinib [10].

While the data from our analyses provide important insights into the potential impact of ethnicity on selumetinib exposure, a number of limitations of the study were identified. Asian and Western PK data were collected from separate studies hence inter-study and temporal confounding variables are not accounted for. The Western PK data were collected from a wide range of healthy subject studies conducted in different sites in the UK and USA, while the Asian PK data were from a single study conducted in the UK. Variability among the methodologies from the data sources limit the conclusions that could be drawn from the pooled data set. Similarly, dose proportionality could only be assessed in a limited dose range (non-Japanese Asian subjects between 25 and $50 \mathrm{mg}$; Western subjects between 25 and $75 \mathrm{mg}$ ) as exposure limits were set for investigations in healthy subjects for selumetinib, again restricting the conclusions that could be drawn from these data. Plasma concentration data was collected for the active N-desmethyl metabolite and PK parameters derived for the respective individual studies. As the proportion of metabolite relative to parent was low and similar proportions were observed across ethnicities, the metabolite data are not reported in the current manuscript.

Finally, the pharmacogenetic analysis had a small sample size, therefore the findings should be considered as exploratory only since these analyses may be underpowered. The possibility that genetic variation at these loci could have an effect on PK parameters cannot be excluded.

In conclusion, this pooled analysis found that selumetinib exposure from single doses of selumetinib was higher in healthy Asian subjects compared with Western subjects in the dose ranges that are clinically relevant. This is important information for clinicians to take into consideration when treating patients of Asian ethnicity with selumetinib.

Acknowledgements These studies and the pooled analysis were funded by AstraZeneca. The authors would like to acknowledge Sandra Brave, PhD, of iMed Comms, an Ashfield Company, part of UDG Healthcare plc for medical writing support that was funded by AstraZeneca.

Authors' contributions All authors fulfilled the ICMJE authorship criteria, including drafting, reviewing or revising the article, and approving the final version to be published. Additionally, the authors were involved as follows: DC, CE, SL, GM, YH: analysis and interpretation of data; UL: substantial contributions to conception and design, acquisition, analysis and interpretation of data; AD, PM, KS; substantial contributions to conception and design, analysis and interpretation of data; HB: acquisition of data. Further, CE conducted the genetic analysis and interpretation of results for the pharmacogenetic component of the analysis; UL was the Principal Investigator on Study 86.

\section{Compliance with ethical standards}

Conflict of interest $\mathrm{CE}, \mathrm{DC}, \mathrm{GM}, \mathrm{YH}, \mathrm{HB}$ and $\mathrm{KS}$ are employees of AstraZeneca, and have stocks/options in AstraZeneca. AD, PM and SL were employees of AstraZeneca at the time of study conduct and have stocks/options in AstraZeneca. UL is an employee of Richmond Pharmacology Ltd. contracted to AstraZeneca $\mathrm{AB}$ to conduct study NCT01960374.

Human and animal rights All procedures performed in studies involving human participants were in accordance with the ethical standards of the institutional and/or national research committee and with the 1964 Helsinki declaration and its later amendments or comparable ethical standards.

Informed consent Informed consent was obtained from all individual participants included in the studies. 
Open Access This article is distributed under the terms of the Creative Commons Attribution 4.0 International License (http:// creativecommons.org/licenses/by/4.0/), which permits unrestricted use, distribution, and reproduction in any medium, provided you give appropriate credit to the original author(s) and the source, provide a link to the Creative Commons license, and indicate if changes were made.

\section{References}

1. Yasuda SU, Zhang L, Huang SM (2008) The role of ethnicity in variability in response to drugs: focus on clinical pharmacology studies. Clin Pharmacol Ther 84(3):417-423

2. ICH (1998) ICH harmonised tripartite guideline. Ethnic factors in the acceptability of foreign clinical data. E5(R1). 1998. Available from: http://www.ich.org/products/guidelines/efficacy/article/efficacyguidelines.html

3. Yeh TC, Marsh V, Bernat BA et al (2007) Biological characterization of ARRY-142886 (AZD6244), a potent, highly selective mitogen-activated protein kinase kinase 1/2 inhibitor. Clin Cancer Res 13(5):1576-1583

4. Banerji U, Camidge DR, Verheul HM et al (2010) The first-inhuman study of the hydrogen sulfate (Hyd-sulfate) capsule of the MEK1/2 inhibitor AZD6244 (ARRY-142886): a phase I open-label multicenter trial in patients with advanced cancer. Clin Cancer Res 16(5):1613-1623

5. Denton CL, Gustafson DL (2011) Pharmacokinetics and pharmacodynamics of AZD6244 (ARRY-142886) in tumor-bearing nude mice. Cancer Chemother Pharmacol 67(2):349-360

6. Ho AL, Grewal RK, Leboeuf R et al (2013) Selumetinib-enhanced radioiodine uptake in advanced thyroid cancer. $\mathrm{N}$ Engl $\mathrm{J}$ Med 368(7):623-632

7. Widemann, B.C., L.J. Marcus, M.J. Fisher et al. (2014) Phase I study of the MEK1/2 inhibitor selumetinib (AZD6244) hydrogen sulfate in children and young adults with neurofibromatosis type 1 (NF1) and inoperable plexiform neurofibromas (PNs). In J Clin Oncol. p. Abstract 10018

8. ClinicalTrials.gov (2015) Study comparing complete remission after treatment with selumetinib/placebo in patient with differentiated thyroid cancer (ASTRA) (NCT01843062). 2015. Available from: https://clinicaltrials.gov/ct2/show/NCT01843062. 11 Dec 2015

9. ClinicalTrials.gov (2016) Selumetinib in combination with gefitinib in NSCLC patients (NCT02025114). 2016 Available from: https:/clinicaltrials.gov/ct2/show/NCT02025114. 07 Feb 2017

10. Oxnard, G.R., S.S. Ramalingam, M.J. Ahn et al. (2015) Preliminary results of TATTON, a multi-arm phase Ib trial of AZD9291 combined with MEDI4736, AZD6094 or selumetinib in EGFR-mutant lung cancer. In J Clin Oncol; 33(suppl; abstr 2509)
11. ClinicalTrials.gov (2016) Single-arm study of selumetinib in combination with docetaxel, in advanced gastric adenocarcinoma patients with low/high MEK signature, RAS mutation or RAS amplification as a second-line chemotherapy (NCT02448290). 2016. Accessed 17 March 2016. 2016. Available from: https://clinicaltrials.gov/ct2/show/NCT02448290

12. ClinicalTrials.gov (2016) Investigate the Safety and Tolerability of AZD6244 Monotherapy or + Docetaxel in Japanese Patients With Advanced Solid Malignancies or Non-Small Cell Lung Cancer (NCT01605916). 2016. Available from: https://clinicaltrials. gov/ct2/show/NCT01605916. 25 Jul 2016

13. Mao Q, Unadkat JD (2015) Role of the breast cancer resistance protein (BCRP/ABCG2) in drug transport-an update. AAPS J 17(1):65-82

14. Li-Wan-Po A, Girard T, Farndon P, Cooley C, Lithgow J (2010) Pharmacogenetics of CYP2C19: functional and clinical implications of a new variant CYP2C19*17. Br J Clin Pharmacol 69(3):222-230

15. Guillemette C (2003) Pharmacogenomics of human UDPglucuronosyltransferase enzymes. Pharmacogenomics J 3(3):136-158

16. Zanger UM, Schwab M (2013) Cytochrome P450 enzymes in drug metabolism: regulation of gene expression, enzyme activities, and impact of genetic variation. Pharmacol Ther 138(1):103-141

17. Marques SC, Ikediobi ON (2010) The clinical application of UGT1A1 pharmacogenetic testing: gene-environment interactions. Hum Genomics 4(4):238-249

18. Zamber CP, Lamba JK, Yasuda K et al (2003) Natural allelic variants of breast cancer resistance protein (BCRP) and their relationship to BCRP expression in human intestine. Pharmacogenetics 13(1):19-28

19. Bosch TM, Meijerman I, Beijnen JH, Schellens JH (2006) Genetic polymorphisms of drug-metabolising enzymes and drug transporters in the chemotherapeutic treatment of cancer. Clin Pharmacokinet 45(3):253-285

20. Dymond A, So K, Martin P et al (2017) Effects of cytochrome P450 (CYP3A4 and CYP2C19) inhibition and induction on the exposure of selumetinib, a MEK 1/2 inhibitor, in healthy volunteers: results from two clinical trials. Eur J Clin Pharmacol 73(2):175-184. doi:10.1007/s00228-016-2153-7

21. Dymond A, Howes C, Pattison C et al (2016) The metabolism, excretion, and pharmacokinetics of selumetinib, a MEK1/2 inhibitor, in healthy adult male subjects. Clin Ther 38(11):2447-2458

22. Dymond A, Martin P, So K et al (2016) Pharmacokinetics of a single oral dose of the MEK1/2 inhibitor selumetinib in subjects with end-stage renal disease or varying degrees of hepatic impairment compared with healthy subjects. J Clin Pharmacol . doi:10.1002/jcph.848.sIn press 